\title{
COVID 19 and Neglected Tropical Diseases in Africa: Perspectives from Ghana
}

\section{Emmanuella Nzeribe ${ }^{1 *}$, Melody Okereke ${ }^{2}$, Isaac Olushola Ogunkola ${ }^{3}$, Mashkur Abdulhamid Isa ${ }^{4}$, Yusuff Adebayo Adebisi ${ }^{5}$ and Don Eliseo Lucero-Prisno $1{ }^{\prime \prime}{ }^{6}$}

${ }^{1}$ Faculty of Pharmacy and Pharmaceutical Sciences, Kwame Nkrumah University of Science and Technology, Ghana

${ }^{2}$ Faculty of Pharmaceutical Sciences, University of Ilorin, Nigeria

${ }^{3}$ Department of Public Health Sciences, University of Calabar, Nigeria

${ }^{4}$ School of Health and Related Research, University of Sheffield, United Kingdom

${ }^{5}$ Faculty of Pharmacy, University of Ibadan, Nigeria

${ }^{6}$ Department of Global Health and Development, London School of Hygiene and Tropical Medicine, United Kingdom

*Corresponding author: Emmanuella Nzeribe, Faculty of Pharmacy and Pharmaceutical Sciences, Kwame Nkrumah University of Science and Technology, Kumasi, Ghana

\begin{abstract}
Since the World Health Organization declared COVID-19 as a public health emergency, the attention of the global community has been shifted towards interventions and efforts to curb the pandemic at the detriment of other diseases, including Neglected Tropical Diseases (NTDs). Prior to the emergence of COVID-19 in Africa, Ghana has recorded some progress in the economy which has translated to an overall expectation of a decline in the incidence of NTDs and other poverty-related diseases in the country. However, the recent COVID-19 pandemic has presented several challenges to the country's fight against NTDs with the potential of reversing the hard-won progress of the country over the years. In this commentary, we examine the impact of the COVID-19 pandemic on NTDs in Africa by sharing perspectives from Ghana.
\end{abstract}

\section{Keywords}

COVID-19, Neglected tropical diseases, Public health emergency, Poverty-related diseases, Ghana

\section{Commentary}

No doubt the pandemic has affected both developed and developing countries in the world [1]. The pandemic added a lot of pressure on the healthcare systems of various countries including Ghana. The country is endemic for a number of NTDs namely Lymphatic
Filariasis, Onchocerciasis, Guinea worm, Trachoma, Schistosomiasis, Soil transmitted helminthiasis, Yaws, Leprosy, Human African Trypanosomiasis (HAT), Buruli ulcer, Cutaneous leishmaniasis and Rabies [2]. Some of the successes recorded in Ghana towards NTDs were a decrease in the prevalence and transmission of onchocerciasis, Iymphatic filariasis, oesophagostomiasis, and yaws, achieved through the combined effects of economic growth and mass drug administration [2,3]. Notably, the country has been overburdened with the high prevalence of these diseases as seen in Table 1 which has received little or no attention over time [3].

Most of the NTDs occurrences were registered in poor settings where access to better sanitation and personal hygiene is a big problem. As a result, poor people who drink water sourced from these areas are at risk of water contamination leading to these tropical diseases [2]. Unfortunately, most of these NTDs are easily preventable, but they remain neglected, thereby, demising so many lives. Although, the mortality rate caused by NTDs is low but it induces ripples of concerns in later stages of lives, leaving many poor people with blindness, cognitive impairment, malnourishment, and stunt in growth, organs deformities, and many other debilitating diseases. Apart from these, stress, fatigue,

Citation: Nzeribe E, Okereke M, Ogunkola IO, Isa MA, Adebisi YA, et al. (2021) COVID 19 and Neglected Tropical Diseases in Africa: Perspectives from Ghana. Int J Trop Dis 4:052. doi.org/10.23937/2643461X/1710052

Accepted: August 26, 2021; Published: August 28, 2021

Copyright: (C) 2021 Nzeribe E, et al. This is an open-access article distributed under the terms of the Creative Commons Attribution License, which permits unrestricted use, distribution, and reproduction in any medium, provided the original author and source are credited. 
Table 1: Showing the situation analysis of NTDs in Ghana based on available data from Master Plan for Neglected Tropical Diseases Programme, Ghana (2016-2020).

\begin{tabular}{|c|c|c|c|c|}
\hline $\begin{array}{l}\text { Neglected Tropical } \\
\text { Diseases (NTDs) }\end{array}$ & Areas Affected & Population Affected & Prevalence Rate & Year of Study \\
\hline Buruli Ulcer & $\begin{array}{l}46 \text { out of the } 170 \text { districts in } \\
\text { Ghana }\end{array}$ & Data not available & Data not available & Data not available \\
\hline $\begin{array}{l}\text { Human African } \\
\text { Trypanosomiasis (HAT) }\end{array}$ & $\begin{array}{l}\text { Five out of the ten regions in } \\
\text { Ghana }\end{array}$ & 4.5 million & Data not available & Data not available \\
\hline Lymphatic Filariasis & $\begin{array}{l}74 \text { out of the } 170 \text { districts in } 8 \\
\text { regions of Ghana }\end{array}$ & 15,000 & $20 \%-40 \%$ & 1999 \\
\hline Onchocerciasis & $\begin{array}{l}40 \text { endemic districts in all } \\
\text { regions except the Greater } \\
\text { Accra Region }\end{array}$ & 2 million & $11.1 \%$ & 2009 \\
\hline Schistosomiasis & 170 districts in all regions & 7 million & $6.3 \%$ & 2008 \\
\hline Soil-Transmitted Helminths & $\begin{array}{l}17 \text { districts in most regions of } \\
\text { Ghana }\end{array}$ & 360,000 & $0.3 \%$ & 2008 \\
\hline Trachoma & $\begin{array}{l}\text { Upper West and Northern } \\
\text { regions }\end{array}$ & 2.6 million & $\leq 10 \%$ & 2000 \\
\hline Leprosy & All districts & 366 & Data not available & Data not available \\
\hline Yaws & All districts in Ghana & Data not available & $0.7 \%$ & Data not available \\
\hline
\end{tabular}

and depression are also linked with NTDs complications $[3,4]$. The government, however, has not relented in efforts to tackle such diseases through several disease specific programmes, utilizing strategies such as case detection and management as well as Mass Drug Administration (MDA). These strategies are in line with the Master Plan for Neglected Tropical Diseases programmes which aims to free Ghana of NTDs and its resulting complications by 2020 [3]. The Master Plan for NTD programme in Ghana is a comprehensive strategic document designed to serve as a guide for all stakeholders executing NTD control/eradication activities with its vision geared towards a Ghana free of neglected tropical diseases and its complications [3].

Quite a number of successes have been recorded in the fight to combat endemic NTDs in Ghana such as Trachoma, Guinea Worm, Lymphatic Filariasis, Onchocerciasis, Human African Trypanosomiasis (HAT) and Yaws [3]. Guinea Worm and Trachoma in Ghana were certified by the WHO as eradicated in 2015 and 2018 respectively while no reported cases of HAT in recent years point to an almost total elimination [4]. Mass Drug Administration with Ivermectin for Onchocerciasis and Ivermectin with Albendazole for lymphatic filariasis has seen a considerable decline in the transmission and prevalence of these two diseases [5]. On the other hand, much still needs to be done to reduce the prevalent rates of schistosomiasis, soil-transmitted helminthiases, Buruli Ulcer and Rabies which are still widespread in rural areas [4]. The government has set and achieved milestones to treat all school aged children once a year against soil-transmitted helminthiases, and in addition, high risk population in endemic parts of the country against schistosomiasis $[3,4]$. Early detection and early treatment measures are being employed to tackle buruli ulcer using the oral antibiotic therapy [5]. With concerted efforts, Ghana seems well-positioned to lead sub-Saharan Africa in the elimination of NTDs [3].

However, these efforts have been grossly disrupted ever since the emergence of the COVID-19 pandemic as all interventions and responses have been directed towards COVID-19 surveillance and management [4]. This current trend and development make it painfully clear that the COVID-19 pandemic has exacerbated the burden of NTDs in Ghana and made interventions focused on addressing NTDs particularly difficult to implement within the context of optimal healthcare delivery [6]. For instance, the symptoms experienced by COVID-19 patients and patients suffering from some of the NTDs such as schistosomiasis are very similar. This can potentially lead to inaccurate diagnosis and treatment in the long term [6]. Also, as a means of curtailing community transmission of the virus, lockdown measures have been implemented in Ghana. While this is a feasible approach of great public health importance, there are concerns that patients suffering from NTDs will face several difficulties in accessing healthcare services due to the lockdown measures. This has been observed in low- and middle-income countries [6] including Ghana.

The onset of the COVID-19 pandemic has overshadowed the continent of Africa with multiple challenges mainly in the healthcare system [7]. Disruptions in health systems have placed a heavy burden on healthcare in many countries [8]. Most hospitals with limited bed space have difficulties in admitting patients with NTD related cases. Drug distributions which in many cases have shown promising outcomes has been suspended resulting in an upsurge of NTD cases. Furthermore, NTD stakeholders have been badly impacted by the suspension of drug distribution due to COVID-19. It is conspicuous that it will cause financial 
losses. Moreover, the presence of clean water, hand and face hygiene are necessary interventions in fight against NTD cases $[5,7]$. However, in many poor countries, this can be a huge barrier to overcome and this is where the government of Ghana intervened by paying water bills for its citizens [9]. NTD programmes received less attention when compared to other diseases. Amidst the COVID-19, it is presumable that poor people inflicted with NTDs, living in poor settings are likely to remain neglected from healthcare facilities due to the paradigm shift in priority to the COVID-19 pandemic [5]. A possible implication is that this will cause unnecessary disabilities later on for many poor people.

\section{Ghana's intervention on NTDs during the pandemic}

As the pandemic progressed, the World Health Organization came up with the various recommendations to deal with neglected tropical diseases during the pandemic era whilst ensuring safety and protection from the virus. Prior to the pandemic, the World Health Organization (WHO) had 5 main strategies in place to end NTDs which are - Preventive Chemotherapy, veterinary public health, vector control, Individual case treatment, and Water, Sanitation, and Hygiene (WASH) [6]. The government of Ghana tackled the WASH strategy by ensuring free water access to all household in the country [9]. Due to the pandemic, the interim guidance was to suspend active case findings while other strategies such as community vector control, veterinary public health, mass administration should only continue with extra precautionary measures in places with no community transmission $[6,10]$. The implication of this led to a hold of some key components of NTDs prevention such as Mass drug administration (MDA) and active screening in Ghana [10]. The country also came up with community information across the borders to train the community health staff on disease control measures [11]. Efforts channeled towards COVID-19 responses have tampered with the progress made towards NTDs elimination but with targeted and scaled-up efforts, Ghana might become the first Sub-Saharan African country with a large populace to achieve extensive success in NTDs control and eradication.

A year into the pandemic, lockdown has been released, social gatherings and schools have resumed with strict measures, it is only logical to have some of the NTD programmes such as the school-based NTD delivery programmes brought back in place with social distancing measures [11]. The fact remains that in a short term, unless the mass drug administration (MDA) and morbidity management activities resume, the achievements of the NTD targets, enhancement of Universal health coverage (UHC) will be deferred and essential medicines will definitely not get to the poorest and the 'leave no one behind' will become a mirage. Further delays on the start of the MDA will cost the country because there will be need for more rounds of
MDA yearly [11]. The key players in combating NTDSthe Sight Savers already published that the inability to complete the yearly MDA will prevent populations from getting access to essential medicines and will definitely leave people behind [12].

\section{Recommendation}

The COVID-19 pandemic had significant impact on the fight against NTDs in Ghana, however, it is paramount that already neglected tropical diseases do not become more neglected. With the non-abating pandemic, innovative ways to resume the NTD programmes ought to be explored after a risk benefit assessment by the government of Ghana. As this is being done, health messaging through the bill boards, radio, TV slots will not only reduce the risk of COVID-19 community transmission but also enhance adherence to the necessary behavior change. The need for partnerships and collaborations cannot be overemphasized in reaching the roadmap of ending NTDs by 2030. Just like every other country, strengthening the capacity of the Ghana health system to match up with the task of curtailing the socioeconomic impact of NTDs in the country.

\section{Competing Interests}

The authors declare no competing Interests.

\section{Authors' Contribution}

The concept for this commentary was developed by NE, MO, 100 and MAI. The manuscript was drafted and prepared by NE and revised by YAA and LEPD. All co-authors read, contributed and agreed with the final manuscript.

\section{References}

1. Lucero-Prisno DE, Adebisi YA, Lin X (2020) Current efforts and challenges facing responses to 2019-nCoV in Africa. Global Health Research and Policy 5: 1-3.

2. Hotez PJ, Biritwum NK, Fenwick A, Molyneux DH, Sachs JD (2019) Ghana: Accelerating neglected tropical disease control in a setting of economic development. PLoS Negl Trop Dis 13: e0007005.

3. WHO (2012) African Regional Office. Master Plan for Neglected Tropical Diseases Programme, Ghana.

4. Molyneux DH, Aboe A, Isiyaku S, Bush S (2020) COVID-19 and neglected tropical diseases in Africa: Impacts, interactions, consequences. Int Health 12: 367-372.

5. Okereke M, Ukor NA, Adebisi YA, Ogunkola IO, Favour lyagbaye $E$, et al. (2020) Impact of COVID-19 on access to healthcare in low-and middle-income countries: Current evidence and future recommendations. The International journal of health planning and management 36: 13-17.

6. World Health Organization (2020) WHO issues interim guidance for implementation of NTD programmes.

7. Blanton RE, Mock NB, Hiruy HN, Schieffelin JS, Doumbia S, et al. (2020) African Resources and the Promise of Resilience against COVID-19. Am J Trop Med Hyg 103: 539-541. 
8. Hopman J, Allegranzi B, Mehtar S (2020) Managing COVID-19 in low-and middle-income countries. JAMA 323 1549-1550.

9. Smiley SL, Agbemor BD, Adams EA, Tutu R (2020) COVID-19 and water access in Sub-Saharan Africa: Ghana's free water directive may not benefit water insecure households. African Geographical Review 39: 398-404.

10. World Health Organization (2021) Neglected tropical diseases: Leveraging the new NTD road map to build back from COVID-19 disruptions.
11. Molyneux D, Bush S, Bannerman R, Downs P, Shu'aibu J, et al. (2021) Neglected tropical diseases activities in Africa in the COVID-19 era: The need for a 'hybrid' approach in COVID-endemic times. Infect Dis Poverty 10: 1.

12. NTD Modelling Consortium (2020) The Potential impact of programmes interruptions due to COVID-19 on 7 neglected tropical diseases: A modelling-based analysis. 